Review Article

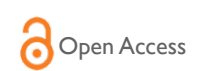

CrossMark

\title{
The special subtypes of déjà vu (part 3)
}

\author{
Volume 2 Issue 6 - 2015
}

\section{Subjective psi déjà vu (section 9)}

\begin{abstract}
I
Subjective psi experience (SPE) is a non-prejudicial term allowing non-prejudicial analysis. The qualitative nosological subtype of SPE déjà vu involves distinct qualities: This grows, is intense, often frequent, and specifically involves time distortions past and future with predictive elements and a specific knowing.
\end{abstract}

Keywords: associative déjà vu, chronic déjà vu, confabulation, continuous déjà vu, definition, déjà, déjà classification, déjà experiences, déjà subtypes, déjà terminology, déjà vu, déjà vu books, history, memory, modern era, multidimensional scaling, neppe, phenomenology, psi, psychodynamic, psychotic déjà vu, redintegration, restricted paramnesia, schizophrenic déjà vu, spe déjà vu, subjective psi experience déjà vu, temporal lobe epileptic déjà vu, tle déjà vu

The view of déjà vu explained by a so-called paranormal experience is the one that holds the most appeal to the layman, but from a purely scientific perspective it is the most extreme of the 72 explanations for the phenomenon - extreme because of its implications, not because of the data supporting it. This is why the author uses the term Subjective psi experience (SPE) so that it can be analyzed non-prejudicially. For a case of déjà vu to be classified as a 'psychic experience', the SPE should be the prime quality. This does not mean it is truly paranormal, just that it is so experienced based on appropriate criteria. However, over many years, accumulating evidence ${ }^{1}$ for at least a subtype of paranormal déjà vu has appeared, based on several researchers and theorists, including the early work of White ${ }^{2}$ in the 1970 s, a prelude to Neppe's $\mathrm{s}^{3,4}$ and Funkhouser's ${ }^{5}$ work in the 1980s.

In some instances, people described their déjà vu while visiting a place for the first time and then successfully predicted what would be found there. For example, people may experience déjà vu as they walk inside an unfamiliar old building, and then astound themselves and their friends by predicting what is in the next room.

A common option might be that the subject previously dreamed about an event and then saw it actualizing. But that would be socalled precognition - foreknowledge. In order to be déjà rêvé, the 'already dreamt' experience, they would either have to have forgotten the dream which was truly precognitive, or more likely rationalize that they must have dreamt it because they could not explain their déjà vu in any other way. That's why the specificity of criteria for SPE déjà vu would have to be very different from an Associative Déjà Vu experience where real forgotten memories or anxieties may stimulate the déjà rêvé and the place or events or persons encountered may appear from an ostensible unforgotten dream. ${ }^{6}$ Or perhaps the sensation of déjà vu may be more complex and have mixtures of the unfolding scenes or events.

Sometimes déjà experiences hypothetically manifest more frequently in what we came to call the 'SPE déjà vu subtype': Thisusually involves 'time distortions', specific subjective paranormal awarenesses, a profound intensity, and a specific predictive "knowledge" with non-psychotic, non-ictal qualities.

\author{
Vernon M Neppe M $^{1,2,3,4}$ \\ 'Director, Pacific Neuropsychiatric Institute and Exceptional \\ Creative Achievement Organization, USA \\ 2Professor, Department of Neurology and Psychiatry, St. Louis \\ University, USA \\ ${ }^{3}$ Executive Director and Distinguished Professor, Exceptional \\ Creative Achievement Organization, USA \\ ${ }^{4}$ Distinguished Fellow of the American Psychiatric Association, \\ USA
}

Correspondence: Vernon M Neppe, Director, Pacific Neuropsychiatric Institute and Exceptional Creative Achievement Organization, Seattle, Washington, USA, Tel 206 527 6289, Email psyche@PNI.org

Received: April 19,2014 | Published: May 26, 2015

Several déjà experiences became relevant in this subtype so are common in Consciousness studies. I regard five as particularly important namely:

1. déjà pressenti: already 'sensed' (as in 'knew' it would happen) (presentiment)

2. déjà retrosenti: already sensed: as a reanimation of living into the past

3. déjà preconnaître: already precognized as well as

4. déjà rêvé: already dreamt (potentially psychological too)

5. déjà vécu: (already lived through, fully experienced / recollected in its entirety)

The following three are less often used:

I. déjà su: already known (intellectually)

II. déjà prevu: already foreseen (not used) (preview)

III. déjà revécu: already lived through or already relived (not used) (reincarnative?)

Let's now take a look at the type of déjà vu reported ${ }^{7}$ by a so-called psychic. Already we can notice the differences in the report compared with the Associative subtype.

"I came to Johannesburg for the first time about six years ago. I had never been there before. I found I just knew how to get to places. I had an impression of knowing the place in detail, as if I had been there before. The experience is ongoing. I still just know my way around. The familiarity sense varies in intensity with my different visits, but generally it is no weaker than my very first experience. Even today I don't use maps.

The section of Johannesburg which is familiar (and was familiar from the very first time) is just the older section. 1 can't find my way around the new suburbs, and buildings or roads built recently are unfamiliar. When I go past, I may say, 'What happened to that building?' I will know that there was something else there before and 
I will feel a little sad that it has come down. Sometimes I can state which building it was. The familiarity sense is strongest opposite the Johannesburg club. There is a block of apartments there. I find it so familiar. I said to my wife, 'It's mine. Maybe I'll buy it someday. 'At times I am aware that certain buildings have been pulled down... I just have a 'knowledge' of certain areas that are very familiar... Time plays no role; I cannot distinguish the past, present or future."

Neppe ${ }^{8-10}$ developed the term 'subjective psi experience' in 1979 $9^{8-10}$ and it has become a key phenomenological concept in consciousness research over the past three decades. ${ }^{10-20}$ In 1981, he coined the term, 'subjective paranormal déjà vu'. ${ }^{3}$ This term has been modified in this article, to the even more neutral 'subjective psi (experience) déjà vu' with 'psi' being used synonymously to 'paranormal'.The concept should be briefly clarified so as to allow a perspective of its relevance. The subject usually describes a sense of marked familiarity with his environment or situation. The experience seems to "grow" as the environment becomes even more distinctive, but the subject remains completely aware of himself. The experience generally occurs frequently and induces both pleasure and a marked sense of conviction. $^{21}$

Applying the model of psychopathology, and we are reminded of the need to talk non-prejudicially. We can refer to subjective hallucinatory experience and be more or less specific: For example, we can talk non-prejudicially about subjective auditory hallucinatory experience or even more so subjective complete auditory hallucinatory experience or subjective auditory pseudo hallucinatory experience. Each has its purpose. This is how DSM criteria in Psychiatry have been developed. It builds upon a phenomenological example (and incidentally, Dr. Neppe has been an advisory consultant for DSM $3^{22}$ and those that followed 3R, 4 and 4TM).

The most striking feature of the experience, however, is that the subject overwhelmingly feels that the sensation is 'paranormal'. The subject is directly aware of a past time and specific facts related to the past. Or he may be able to link the present with direct knowledge of the immediate future: the subject may predict an upcoming event based on his déjà vu.

Again, such descriptions do not necessarily mean that these experiences are genuinely 'psychic'. But such déjà vu is demonstrably qualitatively different ${ }^{3,23}$ from the range of experiences reported by everyday non-psychic people, whose 'associative déjà vu' seems to be vaguer.

This subtype of déjà vu is subjective psi déjà vu (SPE déjà $v u)$. In some ways, it is the most dramatic form of déjà vu. This qualitative nosological subtype of SPE déjà vu involves distinct qualities: This grows, is intense, often frequent, and specifically involves time distortions past and future with predictive elements and a specific knowing.

It seems to occur in two main contexts that have been termed déjà vécu ('already completely experienced' or 'already lived through') and déjà visité ('already visited'). ${ }^{24}$ In a déjà vécu experience, one is living through a situation seemingly for the second time. Everything is exactly as it is 'supposed' to be and one is often convinced that one knows in advance what will occur-and it generally does. It is like seeing a film for the 100th time. Persons having such experiences often remark about the crystal clarity of what transpires and the exquisite detail of what they take in with their senses. At this point in time neither the interpersonal nor the intrapersonal frequencies of déjà vécu and déjà visité in the general population are known with any certainty. The key element is the specific distortion of time: Moving from the present to the past or future.
Some of the déjà experiences may turn out phenomenologically different. For example, when more is known, it could turn out that déjà visité is an entirely different phenomenon. In this experience, a person goes to a place that is new-they are sure they have never been there before - and yet they experience uncanny familiarity with it and the surroundings. But they will describe déjà vécu differently: This 'already lived' experience has to do with reliving a situation, as opposed to the SPE kind of déjà visité which has to do with geographical knowledge that the person is convinced they have no right to possess. As with déjà vécu, precognitive dreams may provide an explanation, but other possibilities for explaining it can include reincarnation and also out-of-the-body travel. Of course, as with Nathaniel Hawthorne, books, films, TV programs, CD-ROMs and other sources of information may be at work here as well.

While seeing may still be the dominant mode of perception, it certainly is not the only one involved, so it is a bit misleading to call this déjà 'vu' (French for 'already seen'), as if only sight was involved. Many, if asked, ascribe such experiences to suddenly remembered precognitive dreams. ${ }^{25,26}$ Because of this link, some SPE déjà $v u$ could easily be linked with the already dreamt experience of déjà rêvé.

This is the key to subjectivity research. We need to use adequate criteria to analyze information. We want to know what we're comparing both qualitatively and at a screening level. We want to evaluate apples as part of the subpopulation of fruits, but we also want to differentiate the vitamin content of South African red apples from Washington green apples.

This is why Funkhouser's recommendation to shorten the term for SPE déjà $v u$ to simply 'paranormal déjà vu' would be inappropriate because we want to be non-prejudicial in our approach and because we want to include all the relevant specificity.

'SPE' is descriptive, neither positive nor negative, but explanatory of a concept. The term 'SPE' is deliberate because as defined it includes the classical categories that are generally alleged to be psirelated. SPE non-prejudicially portrays the subject's impression that they have had experiences of such a peculiar or meaningful kind that they would perceive them as psychic or paranormal or of a psi kind. SPE allows a non-threatening approach to psi, and introduces the phenomenological approach to the broader discipline of the anomalous: The key is the evaluation and categorization of subjective experience so that one can even use subjective anomalous subjective hallucinatory experience if necessary.

The implications of SPE involve another major advance as well. For results to be more meaningful, we need to ensure that descriptions are detailed. By so doing it allows us to avoid making interpretations which are inappropriate: SPE analyses are based on the medical model, and, in fact, much of Neppe's (separate) work on temporal lobe symptoms relies on this same approach. . $2,16,17,27^{2}$

The phenomenological approach adds, rather than subtracts, extra dimensions to evaluations: SPEs that are spontaneous, induced or experimental. When challenged to "prove it happened" or to prove déjà vu, we cannot. Most spontaneous phenomena cannot, by their very nature, be objectively proven. Yet, SPEs permit explorations of such phenomena non-prejudicially. Subjective is used not to negate objective experience. It is a phenomenological way to approach difficult phenomena.

The essential component of subjective psi experience déjà vu is the time distortion. Funkhouser ${ }^{25,26}$ writes about the precognitive element; with many persons, though, there is just as much a retrocognitive 
aspect, distorting backwards in time. This raises a major problem, however: differentiation of what would be precognition that is then retrospectively regarded as an already dreamt experiencefor example déjà rêvé or some other kind of déjà experience at the moment when the person realizes it is being actualized and an actual déjà vu is occurring.

The phenomenological difference is an important one because the familiarity is not inappropriate since it has already been dreamt or already conceived. However, phenomenologically there is no way one can differentiate this. Ostensibly, in our experience, in the majority of instances, the individual has no definite proof or recall that they have actually dreamt that experience or precognized it before in the past. But the differentiation from the subjective psi point of view of those persons where the shift is forward into the immediate futureimmediate precognition - as opposed to backwards into some remote past-remote retrocognition - may turn out to be an important phenomenological difference.

This also is the reason for two terms Neppe has suggested: The first, déjà pressenti, goes back to 1981, implying a presentiment, a déjà type awareness of the actualization of some event moving into the future. ${ }^{3,7}$ The second, déjà rétrosenti, is an invention inspired by the writing of this book in $2006 .{ }^{28}$ Time can as easily move backwards or forwards. However, these terms are not exclusive. Both can be linked with déjà rêvé, déjà visitéand déjà vécu above.

The terms déjà vécu ('already lived through'), déjà visité ('already visited'), déjà pressenti ('already sensed' in the prescience context) and déjà rétrosenti ('already sensed' backward in time) may all reflect overlapping terms for a subtype involving the time distortion, marked familiarity, 'experiential growth', extreme clarity, marked cognitive change, polymodal perception, heightened awareness of the environment, altered awareness of self and usually congruous elevated affective experience with the usually marked sense of conviction and 'sensing' of the experience. These are the features of SPE déjà $v u$.

Thus, this subtype, subjective psi déjà $v u$, also withstands crucial scrutiny. The entity has a distinct place occurring in Subjective Psi Experiments, and has a distinct, unique quality. The pretender term, 'paranormal' - unlike 'subjective paranormal' - might loses the essential phenomenological non-prejudicial character.

\section{Temporal lobe epileptic and brain related déjà vu experiences (section I0)}

\section{Abstract 2}

The relationship of inducing déjà vu through brain firing, including the classical Penfield and Halgren experiments suggest that déjà vu can be inconsistently induced in the brain, more likely in the non-dominant handedness hemisphere and often linked with the temporal lobe but being purely localized. Moreover, later PET studies added to the difficulties of interpretation. When combined with the electrophysiological data beginning with Penfield and moving through to the Guedja work this decade, and adding the Neppe research demonstrating specifically temporal lobe epilepsy déjà vu, Neppe has proposed a provisional explanation model for TLE déjà vu specifically.

\section{Déjà Vu and the brain}

The experimental induction of experiences similar to déjà vu has not only been produced by empirical psychological work in hypnosis or memory distortions in "normal" subjects. Déjà vu can also result from the direct stimulation of the brain's temporal lobe in epileptic patients. ${ }^{29-35}$ The temporal lobe is the part of the higher brainthe cerebrum - that folds under and behind the frontal lobes. The temporal lobe is the great integrator of information, so the locality is not unexpected. Induction of déjà vu by stimulation of the brain strongly supports the 'organic' school's argument that déjà vu reflects an abnormal kind of brain functioning. . $^{30,32}$

But it is very complicated, and the data is incomplete. This is summarized in Table 10A. We begin by illustrating two classic pieces of research.

In 1959, Penfield ${ }^{32,36,37}$ conducted his famous research at the Montreal Neurological Institute..$^{32,35,36}$ Thereafter, in 1978, a group of researchers at the University of California, Los Angeles, reported some related work. ${ }^{33}$ Both research teams were working with temporal lobe epileptics who were undergoing brain surgery performed under local anesthesia. The procedures were implemented so that the surgeons could extract feedback from the patients while stimulating microscopic locations almost always within the temporal lobe.

Penfield found that this form of electrical stimulation sometimes produced déjà vu-like sensations and his patients often relived experiences from their past. Penfield was able to elicit déjà vulike sensations by stimulating association areas in the superior and lateral portions of both temporal lobes in six patients with déjà vu auras. However, he could not elicit déjà vu in four others. The familiarity experiences he could provoke were manly visual, two were not objectrelated and one was auditory. He called such phenomena "illusions of comparison" as they were "interpretations" of the present in relation to the past. ${ }^{30,32}$ Also, Open brain exploration of the temporal lobe and amygdala of the limbic system with micro-electrodes has elicited responses which resemble the familiarity feelings of déjà vu, ${ }^{32}$ indicating a physiological basis for this experience.

The UCLA work expanded these findings, since the researchers there discovered that such experiences could be elicited from the non-diseased temporal lobes of the patients and the exact areas were inconsistent - sometimes even stimulation of one area produces familiarity sensations one time, yet some other experience another time. Even stranger is that another area may then produce these familiarity feelings. The UCLA findings indicate that déjà vu is difficult to locate in any specific area of the brain and is probably not directly related to epilepsy. ${ }^{37}$

Halgren et al. ${ }^{33}$ had available the sophisticated instruments of the modern era to study their 19 patients, in whom they elicited déjà vu in 18. They were able to consolidate Mullan and Penfield's studies by actually delineating which side of the brain was firing by using, among other things, evoked potential responses. They elicited déjà vu in the non-diseased temporal lobe and argued this apparently might not be due to spread of the epilepsy. They also elicited déjà vu in the mesial temporal lobe area, showing the phenomenon to be poorly localizable. ${ }^{33,34}$ The poor localization within the temporal lobe of the phenomenon may also relate to spread of the epileptic focus.

Can interpretations about déjà vu be justified based on neurosurgical stimulation? Apparently it can, but still incompletely. Gloor et al., ${ }^{38}$ in 1981 described a déjà vu experience occurring while an EEG recording was being made. The authors wrote: “... the seizure discharge started in the right hippocampus, then spread to involve the right amygdala and the right parahippocampal gyrus with only modest spread to the right temporal neocortex. It remained confined to the right temporal lobe while the patient described his experiential illusions to the nurse." a

a(pp. 133-4) 
Possibly the most definitive modern localization work belongs to Weinard and co-researchers. They reported a series of eight (amytal tested) left hemisphere dominant patients with ictal déjà vu. Amytal injected into the carotid arteries tests for speech dominance. Subdural strip electrocorticographic monitoring localized the ictal epileptogenic focus to the mesial temporal lobe on the right in six cases and on the left in two. Interestingly, in the six right-handed patients, ictal déjà $\mathrm{vu}$ was associated with a right temporal lobe focus. However, in the two left-handed patients, the ictal focus was in the left temporal lobe. Therefore, ictal déjà vu localizes the epileptic focus to the mesial temporal lobe, but possibly lateralizes to the hemisphere nondominant for handedness, not for speech. ${ }^{39}$

Neppe believes this to be possibly the most important organically based work investigating brain pathophysiology and "temporal lobe epilepsy déjà vu". It provides remarkable insights for the interpretation of sites for related disorders linked with familiarity, reduplication or recollection. ${ }^{40}$ Neppe (previously unpublished) similarly has encountered patients who have had strange phenomenological experiences representing paroxysmal focal temporal lobe abnormalities on push-button during ambulatory EEG. He is, however, reluctant to label these patients as having had déjà vu during this time, in the absence of further detailed phenomenological analyses.

Work has also been also been done this century by Tabet $\&$ Sivaloganathan ${ }^{41}$ who implicated the right frontal lobe close to the midline and possibly the parahippocampus ${ }^{42}$. The works of Penfield, ${ }^{43-45}$ of Halgren ${ }^{33}$ and possibly Weinard, ${ }^{39}$ Gloor and now Neppe have consolidated the theories of déjà vu implicating two kinds of "organic" etiology, namely direct focal firing elements and double aspect hypotheses.

\section{The theories of focal firing, implying the "double-access" hypotheses}

The so-called "double-access" hypotheses from the nineteenth and early twentieth century vary but pertain to a very small delay (eg. in milliseconds) in receiving information which has already been received by another part of the brain. This leads to such information being regarded as having been experienced before when it is processed for the second time. The earliest idea came from Wigan ${ }^{46}$ in 1844. He believed the interval involved to be due to a cerebral hemisphere which was inattentive for a moment. This led Maudsley ${ }^{47}$ to propose asynchrony between the two hemispheres. In contrast, Grasset ${ }^{48}$ felt the interval to be between sensations and perceptions ${ }^{48}$ and Titchener ${ }^{49}$ felt the delay was caused by a momentary disjunction of attentional processes. ${ }^{49}$ The theory that is most commonly cited on this is that of Efron ${ }^{50}$ who believed déjà vu to be due to a delay in receipt of images by the dominant brain hemisphere from the non-dominant one due to a spatio-temporal disturbance..$^{50}$ Another modern idea is that of Comfort, ${ }^{51}$ who believes déjà vu to result from a difference in timing between two major paths of perception causing the one to objectify the other. ${ }^{51}$

Penfield and Halgren's findings, particularly, support the doubleaccess theories, first, because of the distribution of temporal lobe epileptics with déjà vu as an aura-Penfield found nine out of ten of his patents had foci in the non-dominant hemisphere; ${ }^{30}$ and second, because Halgren ${ }^{33}$ was able to elicit déjà vu in nondiseased lobes. ${ }^{33}$ This might increase the chances that déjà vu may be due to the combined working of both diseased and non-diseased hemispheres. Thus, any theory hypothesizing an infinitesimal lag between receipt and processing of déjà vu due to nervous system pathology (either structural or physiological, for example, with fatigue) is indirectly supported.
The era of PET (positron emission tomography) has yielded two important amplifications: Firstly, ${ }^{52}$ in Japan, and his collaborators in London, England, ${ }^{53}$ studied the functional anatomy of the déjà vu experience in nonlesional temporal lobe epilepsy, using interictal fluorine-18 fluorodeoxyglucose PET in 14 patients with reports of déjà vu and 17 patients without déjà vu. Several clinical conditions, such as age at PET study, side of ictal onset zone, and dominance for language, were no different between the two groups. The patients with déjà vu showed significant relative reductions in glucose metabolism in the mesial temporal structures and the parietal cortex. The findings demonstrate that ictal déjà vu is of no lateralizing value. They further suggest that temporal lobe dysfunction is necessary but not sufficient for the generation of déjà vu. Extensive association cortical areas may be involved as part of the network that integrates this distinct experience. However, there is a solvable methodological difficulty, also reflected in all these studies besides small sample size: Neppe has consistently pointed out the problem of analyzing "like with not like". In this study, the researchers did not subtype the TLE déjà vu from Associative déjà vu. It then becomes a problem of heterogeneous data.$^{54,55}$ Later on, Adashi ${ }^{56}$ applied an inventory on déjà vu. (Sno's IDEA $^{57}$ which derives from the Neppe Déjà vu Questionnaire ${ }^{7}$ but it is unfortunate that no inventory was not done in this instance. However, even applying the IDEA is insufficient as there needs to be a clinical side to such evaluations, particularly when studying schizophrenia as he did. It is recommended to use the New Neppe ${ }^{58,59}$ Déjà vu Questionnaire, which is the most updated and comprehensive instrument. ${ }^{58,59}$

Secondly, a similar problem diminishes another otherwise excellent study, this time French in Marseilles, headed by Guedj et al., ${ }^{60}$ and using PET (180-FDG) in TLE patients with and without déjà vu who already had normal Magnetic Resonance Imaging studies of the brain. TLE patients with déjà vu exhibited ipsilateral hypometabolism of superior temporal gyrus and of parahippocampal region, in the vicinity of perirhinal/entorhinal cortex, in comparison either to healthy subjects or to TLE patients without déjà vu $(\mathrm{p}<0$. 05 FDR-corrected). Hypometabolism of both parahippocampal region and superior temporal gyrus was present in $7 / 8$ patients with déjà vu. Hippocampal metabolism was spared in 3 of these 7 patients. Metabolic dysfunction involves a medial-lateral temporal network in patients with déjà vu and normal brain MRI. Within the medial temporal lobe, specific involvement of the parahippocampal region, often in the absence of hippocampal impairment, suggests that the feeling of familiarity during seizures greatly depends on alteration of the recognition memory system.

\section{Clinical research perspectives}

Neppe $^{21}$ has found significant important differences in the way déjà vu was experienced and reported by the different subject populations reflecting the four different nosological subtypes. These make for fascinating reading, but more importantly reflect important lessons in the phenomenology of these experiences. This section gives some brief examples and explanations, and also illustrates the complexity of the descriptions and the analyses particularly in Temporal Lobe Epilepsy. ${ }^{21}$

Neppe demonstrated that the Temporal Lobe Epilepsy déjà vu experience is a special kind that does not occur in patients with other kinds of epilepsy or just a non-epileptic dysfunction of the temporal lobes..$^{7,12,23,61-68} \mathrm{He}$ had postulated that this group of TLE déjà $v u$ patients, seen by neurologists and epilepsy specialists particularly, and uncommon in the general population but not involving a common kind of seizure disorder, would have the typical features linked up with an epileptic aura, and therefore their déjà vu would be 'stereotypical' 
(specific and always the same with some kind of march of symptoms), sometimes unique (because any kind of firing in the temporal lobe may be specific for the patient) and would have so-called post-ictal features like headache, sleepiness and confusion with real perplexity.

Based on the above data, and particularly taking into account the stimulation and PET results, Neppe proposes in this paper that their might be a 72nd explanation for déjà vu but exclusively limited to the Temporal Lobe Epilepsy Déjà Vu subtype: The déjà vu can occur as part of the direct firing, but requires interpretation by the brain using recognition mechanisms. That interpretation is non-dominant hemispheric, producing the impression of familiarity sometimes distorted as an awareness of the difference. The focus may be directly in the non-dominant hemisphere but even when it is not, then nondominant hemisphere for speech or handedness must be involved.

Temporal lobe epilepsy (TLE) déjà vu: The clinical and phenomenological perspective (Section II)

\section{Abstract 3}

The clinical aspects of temporal lobe epilepsy déjà vu based on Neppe's research shows stereotypical "marches" (specific exact progression) of symptoms including post-seizure event features (déjà après), with temporal lobe symptoms and post-ictal features, and an inappropriate familiarity. This makes the diagnosis reasonably easy, but in some instances it could involve paradoxical déjà paradoxe events that potentially may occur under other circumstances.

Neppe has found that there are two main specific kinds of TLE déjà $v u$ namely:

déjà paradoxe 'already paradoxical'; ${ }^{13}$ reflecting the exact déjà differentness being familiar. And déjà après 'already after': After in the sense of the sequence reflecting post-ictal or seizure experiences. These clinical experiences are here matched up with the experimental brain evidence for déjà vu described in the previous section.

Let's examine a typical experience reported ${ }^{7}$ by people suffering temporal lobe epilepsy:

"I have an enormous number of these déjà vu experiences: Up to nine per day for days on end. They always take the same form but the actual details will depend on where I am. While I am having the experience, it is as if I have been there before. This feeling does not occur afterwards when I am coming right. I put these experiences on tape sometimes and I have a big collection of tapes of them."

The same epilepsy sufferer reported this experience:

"I was sitting in the house of a client talking to a customer about a book I was selling. At that moment I got this feeling of familiarity. The whole room was very familiar. What the client asked me that too was very familiar. At the same time, I got the impression of a small river in the house. She saw my whole face was quite white. The whole thing lasted three or four minutes. I continued the conversation as if nothing had happened but meanwhile everything was familiar, and in my thought processes the river inside the room of the house was just there (sometimes it's a river, sometimes it might be chickens). Afterwards, I had a slight headache, and felt tired but not sleepy. This time I was not confused (I sometimes am), and I did not get a rotten egg smell which I sometimes get with it."

Notice that the déjà vu is marked by changes in the subject's thinking and emotional pitch. It is experienced exactly the same way every time, along with heightened awareness of the subject's body and with the environment. Temporal lobe déjà vu can be common or infrequent but the experience is often followed by such side effects as headaches, fatigue, clouded consciousness, blackouts or sometimes, major seizures or other temporal lobe features such as explosive anger, unpleasant odors or other profound sensory distortions.

Neppe re-evaluated all his data a quarter of a century after his pioneering 1981 findings. His findings are empirically based on vast clinical experience with the population of epileptics and patients with temporal lobe dysfunction since 1980 through to 2006, at the time of his trilogy of books after his initial one, ${ }^{7,23,65,69}$ and now through to 2015. Because he has been seeing a specialized population, however, he is reluctant to generalize his findings. Using such questionnaires as his INSET ${ }^{15,70}$ and following up routinely on positive information with detailed clinical enquiry, Neppe frequently elicits déjà vu histories in patients with complex partial seizures and temporal lobe symptomatology. However, working as he has across continents, he does note (until now unpublished) that it is more difficult to elicit histories of temporal lobe epilepsy déjà vu in his current American neuropsychiatric population than it was in his epileptic South African neurological population. Nevertheless, his clinical experience still supports the existence of the separate phenomenological entity of TLE déjà $v u$ associated either as part, or more rarely all, of the complex partial seizure, sometimes with partial status epilepticus (hence, in this rare form, looking like a 'continuous déjà vu'), and less commonly because of the amnesia attendant, with secondary generalized tonicclonic seizures.

He has found that a key feature in individual TLE patients is that their déjà vu experience is often stereotypical; i. e., it is the same every time, it is the same order - the same 'march' of each symptom regularly preceding the next because the same sequence of firing occurs regularly provided it can be recalled. It may be extremely complex, as this classic 'river vision' example shows: ${ }^{7,70}$

"At that moment I got this feeling of familiarity. The whole room was very familiar. What the customer asked me was also very familiar. At the same time, I got the impression of a small river in the house. She saw my whole face was quite white. The whole thing lasted three or four minutes. I continued the conversation as if nothing had happened, but meanwhile everything was familiar, and in my thought process the river inside the room of the house was just there."

The stereotypical sequencing epitomized by such examples led Neppe to propose the term déjà après, ${ }^{28}$ which may be more appropriate than the less precise term of déjà $v u$. Moreover at times there is a sense of distortion best called déjà paradoxe ${ }^{28}$ which he feels is more precise than déjà senti-a non-specific, and not necessarily applicable term which only partly communicates the meaning.

Though there may be stereotypical phenomena with a progressive "march" of symptoms, the déjà paradoxe may reflect literally the paradoxical inappropriate nature of something very simple such as shaving.?

Suddenly I got this feeling that I had done it all before, that this exact thing was being repeated. I can't understand it. Obviously my shaving was being repeated. But it was so familiar, that's what was so strange. 'It was different. '63b

bp4-5

It did not occur every time he shaved. But when it did, it was the same every time.

This may be an example of the depersonalization/ derealization/ sense of strange differentness that the temporal lobe epileptic may 
experience: He knows it's happening all over again, he recognizes it, and yet phenomenologically it is this experience of differentness that creates the inappropriateness. Does the shaving or other event trigger a sequence in the brain, just like redintegration does in the Associative Déjà Vu subject? Will this kind of experience respond to anticonvulsant medication just as the other kinds of TLE déjà vu do? It's a rarity, so it's too early to say. But Neppe's clinical experience has always been that the more controlled the seizure phenomena, the less the TLE déjà vu. Can this kind of déjà vu happen in ordinary individuals? I think it can, but the specificity for this patient was always that it happened while shaving. That potentially initiated the "march" of inappropriate differentness - an exact progression of symptoms, never wavering but potentially stopping if interrupted, for example. For others with TLE, it could conceivably be tooth-brushing, or even orgasmic phenomena and both kinds of seizures have been described (so-called "tooth-brushing induced epilepsy" and "orgasmolepsy"yes, that is a real entity!). Any event that triggers the march can trigger this sense of differentness, provided it then involves the appropriate association areas. This is where the "double aspect" theories can fit in. And yet, for someone with another kind of déjà vu, there may be no stereotypical event, and no specific sequence.

It is likely that TLE déjà $v u$ is sometimes associated with an alteration in consciousness as opposed to the other subtypes of déjà vu that are in clear consciousness. The TLE déjà $v u$ is consistent for the same person: They have the same repetitive 'warning' kind of déjà vu experience. This is termed an 'aura'. So are the post-ictal episodes associated with one or more of following significant symptoms: headache, sleepiness, profound fatigue, disorientation or confused clouding of consciousness, and/or nausea. This stereotypical sequence generally follows the déjà vu aura, hence the term déjà après. ${ }^{28}$ These patients do not have attendant subjective psi experiences (SPEs) ${ }^{12}$ during their déjà vu aura, though they may have them at other times, ${ }^{15}$ nor do they have psychotic misinterpretations. Their experiences are commonly recognized as "happening all over again" but with them comes the sense of inappropriate familiarity, sometimes paradoxically with the depersonalization sense (possibly explaining the correlations found in Heymans and the part replication of Sno), ${ }^{71,72}$ and motivating again the entity of déjà paradoxe. ${ }^{28}$

With regard to the specialized population (epileptics and patients with temporal lobe dysfunction) examined by Neppe for its linkage to temporal lobe epilepsy déjà vu, he summarizes the limiting considerations he has noted: ${ }^{23}$

1. There is some cultural variation of patients admitting these phenomena.

2. There is the need to elicit déjà vu accounts very carefully.

3. Because of the amnesias that are sometimes linked up post-ictally with these phenomena, these patients may not fully remember their déjà vu experiences and therefore there is often underreporting.

4. Neppe does not find TLE déjà $v u$ to be rare. When he assiduously attempts to elicit temporal lobe epilepsy déjà vu from patients with temporal lobe epilepsy, a considerable number of them have déjà vu experiences as part of their simple or complex partial seizure phenomena, but it is difficult to give a figure in this regard without proper large retrospective analyses - and even better, prospective analyses. This is certainly not uncommon and likely resembles the rest of the population.

5. Déjà vu may well be linked up with a cluster of other symptoms pertaining to lateral temporal lobe phenomena. But, because of the specialized sub-populations that he sees, it is difficult to determine exact figures.

Most important, however, was that TLE déjà $v u$ is, indeed, what Neppe called it originally. It is linked specifically with seizures, usually complex partial, and these derive from the temporal lobe. In Neppe's original study, he had a group of 'other epileptics' (not temporal lobe) and their déjà vu was the same as the associative déjà $v u$ of ostensibly normal patients. Thus the use of a term like 'epileptic' déjà vu, which others like Brown ${ }^{73}$ and also Funkhouser have sometimes used, is not only misleading (as only a subtype is involved), but incorrect. ${ }^{3,7,61,70}$ Also, these patients have true seizure phenomena, so a subgroup of non-epileptic temporal lobe dysfunction patients $^{74}$ also has associative déjà vu.

Interestingly, Neppe has been using the Inventory of Neppe of Symptoms of Epilepsy and the Temporal Lobe (INSET) screen and its predecessor the Neppe Temporal Lobe questionnaire for three decades. He has recently examined mesial temporal lobe symptomatology such as explosive outbursts, marked very rapid mood fluctuations, intense episodic fears, olfactory phenomena and reports by others of blanking. He has not noted déjà vu to be part of this cluster, but this is a clinical observation only.

\section{Incidence of déjà Vu in tle}

The incidence of déjà vu in TLE patient is unclear but Neppe argues is at least similar to the general population, and likely more frequent because many also have TLE déjà vu. Neppe ${ }^{75}$ takes issue with the earlier researchers and some later workers who argued that temporal lobe epilepsy déjà vu is a rare phenomenon. ${ }^{75}$ For example, he has discounted the Lennox-Cobb research ${ }^{76}$ as outrageous, where the authors did not provide evidence that they screened for déjà vu and found one case in 750 patients with temporal lobe disease and 4 in 1059 auras! These studies do not reflect appropriate research: They are simply inappropriate, insufficient, non-scientific screening. More latterly, Sengoku et al., ${ }^{77}$ study has similar limitations ${ }^{77}$. Cole \& Zangwill's work ${ }^{78}$ is not much better. Narrow eliciting of incidence by single questions produces far fewer déjà vu in temporal lobe epileptics, as evidenced by the published literature.

When one re-examines the data when adequate screening questions are asked, using Neppe's ${ }^{3-7}$ and Sno's, ${ }^{57}$ instruments the incidence of déjà vu in temporal lobe epilepsy suddenly resembles the general population at $74 \%$. Furthermore, the TLE déjà $v u$ experience is different and possibly in the same range of commonality as normals. It will, of course, be a little less frequent when the incidence is based on one question or sometimes no questions! TLE déjà $v u$ needs to be specifically screened for.

In summary, some temporal lobe epileptics have a significant amount of déjà vu events. Based on the data available, these appear ostensibly inversely proportional to the degree of control of their seizures.

\section{Clinical perspective}

There is a specific common phenomenological similarity to patients with temporal lobe epilepsy and their post-ictal and related ictal phenomena. They will have post-ictal headache, sleepiness and confusion afterwards, not déjà vu per se. And there could be a location most likely in the mesial temporal lobe in the non-dominant hemisphere for handedness. ${ }^{39}$

Sometimes the aura of a seizure (and that might be what the déjà vu could be in temporal lobe epilepsy) is not recalled because 
of amnesia. Then any déjà vu may not be remembered. Ironically though, patients frequently will describe the déjà vu they have even years later because when recalled it is a profoundly impacting experience. Conversely, commonly the déjà vu experience, as well as the aura, is remembered.

This raises a core question: Is there then a danger of TLE déja $v u$ actually not being déjà $v u$ because the familiarity is perfectly logical — the subject is expecting his aura and recognizes it as familiar? That's why we must quantify. We define what is meant by TLE déjà $v u$. If it fits, then it's $T L E$ déjà $v u .^{40}$ Indeed, it will only fit if the actual aura itself experienced as appropriately familiar because it is the same as in previous seizures, is accompanied by a sense of inappropriate familiarity(hence, Funkhouser had suggested the term 'déjà senti' to reflect this 'already sensed' component. ${ }^{79}$ The key features essentially are stereotypical links with other possible temporal lobe symptoms, full-blown seizure phenomena with impaired consciousness or loss of consciousness, or post-ictal features. There is also no reason why a patient with TLE but other kinds of auras should not have a familiarity with what is coming next-but that is not déjà vu, because the familiarity is appropriate.

The Neppe ${ }^{61}$ unification was to demonstrate that the subtype of patient with TLE has specific qualitative events. When they are uncontrolled, the stereotypical marches - the ordered, regular and invariable progression - of one déjà vu may be superimposed upon another or closely linked. This could create a situation where such phenomena appear as continuous. There may even be complex partial déjà vu status epilepticus conceivably with one déjà vu seizure aura episode superimposed on another. But this reflects poor seizure control, not continuous déjà $v u$. This may be why the lifetime incidencethe number of subjects per 100 having any déjà vu at any point in their life - of déjà vu in TLE may be similar to the general ostensibly normal population, even while the frequency in specific individual patients of their déjà vu may vary widely and early reports always referred to how patients with TLE had frequent déjà vu. ${ }^{61}$

Neppe does, however, note an overlap in this population. There is no reason why any epileptic patient may not in addition or instead have common-garden associative déjà $v u$ independent of any aura. If the qualitative features do not fit, that is not TLE DV. Neppe particularly has seen numerous cases of associative déjà $v u$ (the 'normal' déjà $\mathrm{vu}$ ), no different from the general population, in all kinds of epileptic patients. The one does not exclude the other. The associative déjà $v u$ complex of events still remains the common-garden variety déjà vu.

Moreover, because of the demonstrated correlation of temporal lobe disorders and subjective psi experience - another of Neppe's research contributions in the area ${ }^{12,16,17}$-the theoretical occurrence of TLE déjà $v u$ and SPE déjà $v u$ in the same person is possible. Technically, SPE déjà $v u$ as well as TLE déjà $v u$ should occur in TLE patients who have SPEs, but strangely we have not seen both subtypes in the same patient. Nor have we seen the combined occurrence of the features that constitute SPE déjà $v u$ with those features of TLE déjà $v u$ in the same patient. Thus this complex phenomenological kind of déjà vu is yet to be described or reported.

\section{Psychotic déjà Vu (section I 2)}

\section{Abstract 4}

Schizophrenic (Psychotic) Déjà Vu is examined based on the Neppe nosological subtype. It is like Associative Déjà Vu, but has psychotic features, often elicited through the déjà vu questionnaires and including thought disorder, self referential ideas and special meanings. This leads to the possible use of the Neppe Déjà Vu Questionnaires as a structured evaluation for psychosis. Psychotic déjà vu seems to uniquely occur in psychotic patients and is phenomenologically distinct.

Neppe proposed and found there was a special kind of déjà vu linked with psychotic thought. ${ }^{7,66}$ Patients with this kind of déjà vu were isolated to the schizophrenic subgroup, and, like the other three subpopulations, were distributed in one of the four separate quadrants when analyzing phenomena applying median column geometry in 22 dimensions. Initially, he analyzed only relative stable schizophrenics, but clinically and very provisionally, he has found this occurring in bipolar patients, as well as confusional states.

In many features, this kind of déjà vu has remarkable similarities to Associative Déjà Vu. But the difference is that there is generally no perplexity because everything is obvious and explained by the patient by thought disorder, misinterpretation of reality, illogical ideas pertaining to self, overt psychotic features sometimes with auditory hallucinations, and lack of insight. These are all prototypical features of schizophrenic psychosis. Even more remarkable, the subjects analyzed in the original Neppe study ${ }^{3}$ were not floridly psychotic, yet the Neppe Déjà Vu Questionnaire amplified by a clinical interview was able to elicit psychotic thought. The patients generally enjoyed talking about these idiosyncratic ideas because they did not feel threatened and this therefore can potentially be used as a method to elicit psychotic thinking in patients who may or may not be overtly psychotic. This diagnostic use has not yet been applied but has enormous potential in my opinion. I would certainly recommend the application of Neppe's structured Revised Déjà Vu Questionnaire as a projective test in schizophrenia as a possible $\mathrm{PhD}$ thesis.

Two kinds of déjà experiences appear prevalent, at times, in Psychotic Déjà $\mathrm{Vu}$ :

a. déjà ésotérique: already esoterically perceived: with special significances often psychotic

b. déjà halluciné: already hallucinated: I've hallucinated this before-an intriguing experience! But this is not specific: strangely, it has been elicited even more in acute delirium.

And a third is purely theoretical, as I do not know of a single case description:

c. déjà voulu: already desired already wanted could be but likely would not be a rare part of a delusional system.

Although Neppe's,65 original study involved schizophrenic patients, ${ }^{7,65}$ he is uncertain whether he should better call this subtype Psychotic Déjà $\mathrm{Vu}^{23}$. As official studies have yet to be done in other psychotic conditions, such as in bipolar disorder, it is uncertain whether this nosological subtype can be extended to other forms of psychosis. But Neppe has officially changed the name from his original schizophrenic déjà vu to psychotic déjà vu..$^{23}$

Sometimes, there are bizarre misinterpretations of reality linked up with the sense of vague inappropriate familiarity that is found in schizophrenia—psychotic déjà $v u$. The psychotic features relate to thought disorder, self-reference, and special meanings - all common features of poorly controlled schizophrenic thinking. However, psychiatric nomenclature is at this point so complex and in so much flux that I believe it is appropriate to accentuate the psychotic phenomenology here, without using a label such as 'schizophrenic'. 


\section{The following is an example taken from a schizophrenic patient: ${ }^{7}$}

"Once I saw photos of Israel where Jesus was born. It showed the crib, and the star. I felt very significant feelings. It did something for my mind. I had a warm feeling. I felt I was near home. I felt I had been there before a long time ago - centuries ago - at the time of Christ. Sometimes I feel I'm an eternal spirit, Socrates, Churchill."

Now remarkably, the patient had not exhibited any formal thought disorder. He was what Neppe as a psychiatrist who specializes, inter alia, in mental status assessment, until then, regarded as 'relationally apsychotic'.

While filling out the déjà vu questionnaire, the subject said he believed he was Christ and had been crucified. This is classically what Neppe ${ }^{21}$ has termed 'psychotic déjà vu'. It is distinguished by such psychotic interpretations. These subjects tend to overplay their experiences with such bizarre, illogical thinking. ${ }^{21}$ The psychotic misinterpretation of reality involves the déjà vu being part of a more intricate distortion of information or events which have a peculiar, even idiosyncratic, meaning of special significance for the person experiencing it. ${ }^{23}$

Remarkably, in Neppe's original study, these non-floridly psychotic schizophrenics showed evidence of psychotic features in all 13 subjects with déjà vu, and, in fact, thought disorder in at least minimal form was elicited via the screening questionnaire even in subjects not admitting to déjà vu in all but one case. Thus 95\% (19/20 subjects) had at least minimal thought disorder and in $75 \%(15 / 20)$ there was definite thought disorder. This finding achieves greater perspective in that no other subjects in any other group (both statistical-i. e., conforming to the stipulated sampling criteria - and non-statistical-i. e., analyzed as special cases) without previous history of psychosis ( $\mathrm{N}=66$ ) exhibited even minimal thought disorder. This again supports the application of Neppe's structured questionnaire as a projective test in schizophrenia. ${ }^{7,65}$

As described in his original collection of cases, traditionally many of Neppe's psychotic patients with déjà vu have short-standing déjà vu frameworks as part of their delusional phenomena. ${ }^{3,7,37,70,80}$

These Schizophrenic patients acted as an adequate comparison group to the TLE patients. Although the déjà vu experiences in the schizophrenics had previously been poorly investigated, the hypothesis was that certain special features might exist in their experiences representing the 'psychotic prototype'. In all other subtypes there was already far more support in the literature, but not in 1979 for Schizophrenic or Psychotic Déjà Vu. These were unchartered waters. ${ }^{7,65}$

as special meanings, delusional awarenesses, and vague collective unproven knowledge which serves as a spring board for misinterpretation of the environment. ${ }^{7,65}$

Psychotic déjà $v u$ is qualitatively specific:

a. There is not the perplexity of the associative déjà vu; rather, there is the knowing certainty. There is also generally not the attribution of the déjà vu to some event or place.

b. There is also not the specificity of time distortions of SPE déjà $v u$.

c. There are neither the post-ictal variations, nor the complexity of other symptoms that are sometimes linked with the symptoms of temporal lobe epilepsy (what Neppe calls "possible temporal lobe symptoms") 12,16,17,27,81,82 that constitute TLE déjà vu.
Interestingly enough, the déjà vu experiences in psychotic déjà $v u$ are sometimes the only visible sign indicating mental illness. Because of that, déjà vu experiences can be a tool in diagnosing mental illness. Clearly, such diagnoses are also based on impairments in functioning and coping at the ethico biopsycho familio socio cultural level. ${ }^{83,84}$ Neppe has also emphasized that psychotics will tolerate antipsychotic doses of neuroleptics - biologically they are different. ${ }^{82-84}$

The key to psychotic déjà $v u$ subcategory, however, is the psychotic element. Otherwise, psychotic déjà vu may look like vague associative déjà $v u$ with the added, at times unauthenticated, vague SPEs like "I know what will happen", but with nothing concretely distinctive. Neppe ${ }^{3,7}$ feels that this impression of vagueness is also, at times, an inappropriateness. ${ }^{3,7}$ Schizophrenics, however, do not commonly describe a sense of perplexity. Instead they hook what 'normals' would regard as perplexity into special meanings- they delusionally may interpret what others perceive as inappropriate, as a special message or awareness. These are then fitted into frameworks that are meaningful - and therefore appropriate-within their psychosis.

Some patients with psychosis are careful about verbalizing psychotic thought and this may trigger defensiveness. However, when describing their déjà vu, their guard sometimes comes down and, as they lack the insight that they are ill, they may describe thought disorder, special meanings and delusional ideation which they would not generally admit to because of their paranoid overlay.

$\mathrm{Neppe}^{3,7}$ suspects that the non-threatening and interesting description of one's personal déjà vu is a core reason why déjà vu can be used as a projective test in the psychotic patient. This projective testing relates particularly to the Déjà Vu Questionnaire (DVQ). ${ }^{3,7}$ This is so non-intrusive that schizophrenic patients do not feel threatened and are prepared to describe their déjà vu freely. This in turn produces a fertile environment for them to talk about their delusions, their hallucinations, the abnormal links of their thought processes, and the irrelevant misinterpretations. Individuals who are too psychotic cannot communicate information about déjà vu. On the other hand, patients whose thought disorders are under control may not manifest déjà vu at all. Déjà vu may, however, be a useful projective test to elicit disordered cognition in the group in between these extremes. Neppe has seen non-florid, well-defended psychotic patients whose thought disorder comes through when administered this structured Déjà Vu Questionnaire in a non-invasive conversational manner.

Psychotic déjà $v u$ may be ongoing with misinterpretations but that is less likely (just as TLE déjà vu may be). More commonly, once patients are treated with neuroleptics they do not have these experiences. This is therefore not continuous, and even during psychotic phases there is only a window at which time the psychotic déjà $v u$ manifests.

The occurrence of déjà vu among temporal lobe epileptics and schizophrenics still appears qualitatively different from that of the general population with specific subtypes of déjà vu and different from each other. This means we are not apparently only dealing with patients who suffer from one or the other ailment and who also happen to have déjà vu experiences, but with patients who have their own special diagnostic qualities to their déjà vu. Schizophrenic déjà vu occurs in schizophrenics, just as temporal lobe epileptic déjà vu occurs in Temporal lobe epilepsy and no other kind of seizure disorder or non-epileptic temporal lobe dysfunction., ${ }^{7,65}$ 
In our experience, many schizophrenics will not conceive of déjà $\mathrm{vu}$ in the (associative déjà $v u$ ) context in which so-called normal individuals usually experience it. Consequently, it is actually more difficult to elicit the phenomenon, unless it is broadly screened for. However, once elicited, the bizarre associations and special meanings manifest.

In conclusion, there remains an entity called psychotic déjà $v u$, which seems to uniquely occur in psychotic patients and which is phenomenologically distinct.

\section{Continuous and chronic déjà Vu:a new entity? (section I3)}

\section{Abstract 5}

Confabulation and recollective confabulation are examined in the context of organically impaired individuals with fronto-temporal disease. Rarely, Continuous (often Chronic) déjà vu occurs though whether these cases conform to déjà vu is questionable. Such chronicity can also occur in normals, temporal lobe epilepsy déjà vu and in psychotic déjà vu.

They are a phenomenological challenge.

\section{Confabulation is not déjà Vu}

Confabulation is a well-known organically based memory disorder in which the patient will fill in the gaps of a past experience, very often a recent past event such as what he or she did that morning, by effectively providing what seem to him or her to be-but are notlegitimate explanations for such events. The patient may describe the bacon and eggs eaten for breakfast and the visit by his son. However, none of that may be true. There is no sense of familiarity, just a false recollection of the past. ${ }^{7,44}$ Classically, confabulation is associated with a neurologically based memory disorder called Korsakoff's syndrome, and this in turn, may be linked with such causes as alcohol excess or brain anoxia. ${ }^{45}$ Confabulation becomes relevant for déjà vu, given the new spin which the British psychiatrist, Dr Chris Moulin and his coworkers have given it, with the term recollective confabulation. ${ }^{85}$

\section{Recollective confabulation: déjà vu vs. déjà} vécu or a separate entity?

Dr. Moulin ${ }^{86}$ and his colleagues point out how some cases may not be recognized as cognitive disturbances indicating brain pathology. Moulin et al., ${ }^{86}$ introduced the concept of recollective confabulation involving dysfunctional recollection in memoryimpaired subjects who recognize their impairment, but nevertheless act on their experience. They confabulate an explanation, usually as a false memory that accounts for, or justifies the feeling. Because of their continuous false recollections, patients confabulate to justify.

"the impression they have that they have encountered the present moment before and they fail to control and monitor these erroneous sensations of remembering". 86

Moulin et al., ${ }^{86}$ described two cases in patients with dementia and diffuse temporal lobe pathology with what he is regarding as repetitive déjà vécu, in that they felt have lived through (or certainly recollected) the present moment before. The descriptions, at times, apparently conform technically with the standard Neppe operational definition of déjà vu-namely, any subjectively inappropriate impression of familiarity of a present experience with an undefined past. ${ }^{7}$ Neppe feels that, if these are, indeed, déjà experiences, the events described would be more correctlytermed déjà pensé, as they really are more 'already thought' experiences, as well as déjà vécu, if this includes 'already recollected' as opposed to exclusively 'already lived' experiences.

Cases such as these are important as they challenge the conventional déjà vu definition (or déjà vécu as a subtype of déjà vu). However, an important difference here is that the impression of familiarity is not technically of the present experience but of the present thoughtbut déjà pensé could cover that. However, Moulin et al. state:

"In contrast, our patients are anosognosic for their inappropriate sensations." " ${ }^{86}$ However, to both Neppe and to Funkhouserc, there remains the question of whether these events describe déjà experiences at all. This is so as, by definition, déjà vu requires inappropriate familiarity with the present '. ${ }^{3}$ Thereafter this leads to consequential reactions. For example, in Associative déjà vu, the bewilderment about the inappropriateness; in SPE déjà vu subtypes, an awareness of the illogicality and subjective experiences that follow on this; in TLE déjà vu, the symptom march or post-ictal features or impression of unreality or differentness; in Psychotic déjà vu, the insightless continuation of some kind of delusional awareness or hallucination or thought disorder as a consequence of the déjà experience. With Moulin's cases, there appear to be no responses ${ }^{87}$ There is purely the impression of familiarity of the present with a sometimes undefined, possibly forgotten past: There is no illogicality or rationalization or startled reaction or perplexity: it just is. Any reaction is not a consequence of the possible déjà event: Moulin portrays the experience as 'recollective confabulation' ${ }^{87}$ But confabulation, as pointed out, is not déjà vu. This means that the patients effectively do not recognize the inappropriateness. This makes such experiences not déjà vu nor déjà vécu, because the impression of inappropriate familiarity is a key part of the definition of déjà vu. The Moulin contribution can be fruitfully analyzed in the context of related research and the phenomenological application of fundamental terminology.,

cNeppe and Funkhouser in discussion April 2015.

\section{Continuous and chronic déjà Vu}

Because there are those such as Moulin et al. ${ }^{86}$ and Funkhouser ${ }^{79}$ who write about this kind of déjà vu, the entity of 'Continuous Déjà $\mathrm{Vu}$ ' is here mentioned. This pervasive group of schizophrenics theoretically could have frequent déjà vu, and when very uncontrolled could be 'continuous'. This would mean that just as the floridly, chronically psychotic schizophrenic could be almost continuously hallucinating while awake, they could have 'Continuous Déjà Vu'. In my opinion, based on evaluating thousands of schizophrenic patients, this is theoretically possible but unlikely to be so severe. Thus, the essence of psychotic déjà $v u$ is not whether it is usually continuous or maintained. Some psychotics may have repetitive delusional thinking until controlled, but continuous is not the key feature: It is the thought disorder that is key in psychotic déjà $v u$. Thought disorder is one key to the diagnosis as to whether the person is psychotic or not.

Extending this concept of 'continuous', the continuous framework of déjà vu might not only reflect psychotic déjà $v u$ per se, but the temporal lobe epileptic type déjà vu with continuous déjà vu status epilepticus or numerous seizures per day. In fact, Neppe ${ }^{87}$ describes one such a patient whose déjà vu responded to anticonvulsant medications. ${ }^{87}$

Is Funkhouser's proposed subtype of continuous déjà vu a new entity? ${ }^{40}$

The rationale for 'continuous' might be a good one: ensuring that labels become non-prejudicial and avoiding necessarily pathological 
connotations. Theoretically, this continuous form of déjà vu might go on for months but because hypothetically, it does not imply either continuous psychosis or seizures, but may be a chronic variant, for example of Moulin's Alzheimer group 'chronic' may be more accurate.

Funkhouser believes the salient feature of the continuous type is that it lasts for a long time (i. e., months or even longer) and the person believes that he or she is actually reliving a portion of their life. They claim to recognize everything that happens as being familiar. There is nothing new for them. He has described one (unpublished) case of an ostensibly 'normal' individual which therefore could justify the 'continuous' label. One could say that their familiarity 'switch' is permanently in the 'on' position.

On the other hand, in all these cases (the one normal case, schizophrenia, temporal lobe epilepsy, Alzheimer's disease), there does not seem to be a predictive dimension to it- they are apparently unable to say what is going to happen next.

This may complicate interpretations even more: Such an individual may become so rattled by this state of affairs that they become mentally ill and become placed under psychiatric care. This psychotic form of déjà vu has also been termed 'reduplicative paramnesia ${ }^{87}$ and later as 'chronophrenia'. ${ }^{88}$

Neppe points out the significant dispute as to whether any of these pervasive, chronic or continuous cases even justify the definition label of déjà vu, and if they do, it may be only in a proportion of the cases reported. ${ }^{28,89}$

Neppe $^{40}$ argues for a spectrum of 'déjà continuity' throughout all our four déjà vu nosological subtypes. The spectrum in all four subtype conditions could run from occasional through to extremely frequent, and possibly rarely to the very few who incessantly maintain it is happening constantly. ${ }^{40}$ 'Continuous déjà vu' to Funkhouser and occasionally to Neppe ${ }^{\mathrm{d} 23}$ (in the past), is a better term than 'Chronic déjà vu'. which Brown ${ }^{90}$ and Moulin ${ }^{86}$ might prefer, as it does not imply necessary pathology, but I currently think that if such an entity exists, it is more likely to be more characterized as chronic not continuously.

\section{dP42}

Importantly in this regard, Neppe has described one case of complex partial seizure status epilepticus involving profound clusters of tens of episodes daily manifesting purely as déjà vu; the subject was diagnosed with complex partial seizures and dramatically responded to carbamazepine. In fact, the theme of anticonvulsant responsiveness in such cases may obviate the need for a further category. ${ }^{88}$ Until such subjects receive comprehensive neuropsychiatric evaluation to establish whether they are having uncontrolled simple or complex partial seizures, or possible psychosis, Neppe remains disinclined to accept the expansion of continuous déjà $v u$ to include ostensibly normal déjà vu.

To re-iterate: Although Neppe may agree that not all subjects may be covered by his strict definitions of his four fundamental subtypes, this does not obviate the need to have good supporting data to justify the inclusion of new subtypes or the subdivision of existing subtypes.

Conversely, we must be careful to label cases that don't even fit the basic definition criteria of particular subtypes. This highlights the need for a qualitative analysis in detail whenever new data challenge our fundamental framework for déjà vu.

At this point, Neppe cannot yet justify a fifth subtype called continuous déjà vu. It has no nosological specificity-it does not occur in a particular population-and it has no special, phenomenologically unique qualities in its déjà vu itself. Moreover, it has not yet been proven to exist.

\section{Perspectives}

Neppe's main theoretical conclusion of this review merits quotation in full:

Déjà vu is the epiphenomenon underlying numerous different phenomenologies. This would imply that Temporal Lobe Epileptic Déjà $\mathrm{Vu}$ is associated with a specific pattern of cerebral firing, that Schizophrenic Déjà Vu is symptomatic of the underlying reality distortion, that Subjective psi experience Déjà Vu should be classified as a kind of subjective psi experience with heteropsychic origins, and that Associative Déjà $\mathrm{Vu}$ is due to a redintegrative restricted paramnesia mechanism, possibly "learnt" as an unconscious reassurance-type ego-defense.

Some argue that the most enduring facets of research survive a quarter of a century and much longer. Looking at the déjà vu phenomenon, theories still continue to abound, although they still fit within the frameworks of the Neppe model of $1981 .{ }^{7}$

Importantly, what has not changed is Neppe's fundamental definition of déjà vu:

"Any subjectively inappropriate impression of familiarity of the present with an undefined past."

The four elements remain reflecting the four major subtypes:3,7

i. psychophysiological and psychodynamic associations of normal cognitive brain function,

ii. abnormal temporal lobe firing,

iii. psychotic preoccupations, and

iv. subjective psi experiences.

These entities are unique, distinctive and fit within distinctive diagnostic (nosological) categories. They therefore fulfill the criteria to be so classified. The fifth, déjà vu in the Alzheimer perspective be it labelled 'Chronic' or 'Continuous' remains under debate.

What has also not changed is the differentiation of these four specific phenomenological subtypes developed to account for and organize the diversity manifested as 'déjà experience'. However, what has changed is the extension now to 36 different déjà experiences, besides the twenty-one kinds originally described by Neppe in his 1983 book. ${ }^{7,28}$ This durable framework is pivotal for the integrity of the subtype classification as well as of the distinctive elements occurring in these four very different population subtypes. These categories suggest not only phenomenological specificity, but likely different causalities and a need to be aware that déjà vu is not based on a single explanation. ${ }^{7}$

\section{Acknowledgments}

None.

\section{Conflicts of interest}

Author declares there are no conflicts of interest.

\section{Funding}

None. 


\section{References}

1. Halgren E, Chauvel P. Experimential phenomena evoked by human brain electrical stimulation. In: Devinsky et al. (Eds.), Electrical. 1993.

2. White RA. The mystery of deja vu: Who knows where or when? Psychic. 1973;4(5):44-49.

3. Neppe VM. A study of déjà vu experience: thesis. Unpublished doctoral thesis, University of the Witwatersrand, Johannesburg. 1981.

4. Neppe VM, Déjà vu. A parapsychological approach. In: Roll WG et al (Eds.), Metuchen, Research in Parapsychology 1982. Scarecrow Press, USA. 1983. p. 226-228.

5. Funkhouser AT. Deja Vu: Deja Reve. Unpublished diploma thesis, C.G. Jung Institute, Küsnacht, Switzerland. 1981.

6. Funkhouser AT. Dreams and déjà vu: chicken or egg? Déjà Vu: A Second Look (Chapter 10). Brainvoyage.com. Seattle, USA. 2006.

7. Neppe VM .The Psychology of Deja Vu: Have I been Here Before? Johannesburg: Witwatersrand University Press. 1983

8. Neppe VM. Subjective paranormal experience. Psi. 1980;2(3):2-3.

9. Neppe VM. An investigation of the relationship between subjective paranormal experience and temporal lobe symptomatology. University of Witwatersrand, Johannesburg. 1979.

10. Neppe VM. A study of the incidence of subjective paranormal experience. Parapsychological Journal of South Africa. 1981;2(1):15-37.

11. Neppe VM. Anomalies of smell in the subjective paranormal experiment Psychoenergetics - J of Psychophysical Systems . 1983;5(1):11-27.

12. Neppe VM. Temporal lobe symptomatology in subjective paranormal experients. Journal of the American Society for Psychical Research. 1983;77(1):1-29.

13. Neppe VM.Anomalous smells in the subjective paranormal experient Psychoenergetic Systems . 1983;5:11-28.

14. Swiel DJ, Neppe VM. The incidence of subjective anomalous experience in naive subjects. Parapsychological Journal Of South Africa. 1986;7(1):34-53.

15. Palmer J, Neppe VM.Subjective paranormal experiences and temporal lobe dysfunction in a neuropsychiatric population: analyses of refined predictors. Journal of Parapsychology. 2003; 67(1):75-98.

16. Palmer J, Neppe VM. Exploratory analyses of refined predictors of subjective ESP experiences and temporal Lobe Dysfunction in a neuropsychiatric population. European Journal of Parapsychology. 2004;19:44-65.

17. Palmer J, Neppe VM. A controlled analysis of subjective paranormal experiences in temporal lobe dysfunction in a neuropsychiatric population. Journal of Parapsychology. 2003;67(1):75-98.

18. Swiel DJ, Neppe VM. The incidence of subjective anomalous experience in naive subjects. PJSA. 1986;7(1):34-53.

19. Funkhouser AT, Neppe VM. Update on the incidence of déjà vu: an appendix. In: Neppe VM \& Funkhouser AT (Eds.), Déjà Vu: A Second Look (Chapter 7b). Brainvoyage.com. Seattle, USA. 2006. p.79-91.

20. Neppe VM. The incidence of déjà vu. Parapsychological Journal of South Africa. 1983;4(2):94-106.

21. Neppe VM. Illustrative Examples of déjà vu in the four different subtypes (Section 7). IQNexus Journal. 2015;7(1):47-50.

22. Committee APA. Diagnostic and Statistical Manual: DSM 1V-TR (4th edn). American Psychiatric Association, Washington D. C, USA. 2000

23. Neppe VM, Funkhouser ATs. Déjà Vu: A Second Look. Brainvoyage. com. Brainquest Press, Seattle, USA. 2006.
24. Funkhouser AT. Three types of déjà vu. Scientific and Medical Network Review. 1995;57:20-22.

25. Funkhouser AT. The "dream" theory of deja vu. Parapsychological Journal of South Africa. 1983;4(2):107-123.

26. Funkhouser AT. Dream Theories of Déjà Vu. Dream Network 2004;23(3):15-17.

27. Neppe VM. Symptomatology of temporal lobe epilepsy. S Afr Med J. 1981;60(23):902-907.

28. Neppe VM. Does the definition of déjà vu withstand a quarter of a century of research? In: Neppe VM \& Funkhouser AT (Eds.), Déjà Vu: A Second Look (Chapter 15). Brainvoyage.com. Seattle, USA. 2006.

29. Penfield W, Boldrey E. Somatic motor and sensory representation in the cerebral cortex of man as studied by electrical stimulation. Research publications association for research in nervous and mental disease. 1937;60(4):389-443.

30. Penfield W. Functional localization in temporal and deep Sylvian areas. Res Publ Assoc Res Nerv Ment Dis. 1958;36(1):210-226.

31. Penfield W. The role of temporal cortex in certain psychical phenomena. Journal ment science . 1955;101:451-465.

32. Mullan S, Penfield W. Illusions of comparative interpretation and emotion. AMA Archives of Neurology and Psychiatry. 1959;81(3):269-284.

33. Halgren E, Walter RD, Cherlow DG, et al. Mental phenomena evoked by electrical stimulation of the human hippocampal formation and amygdala. Brain. 1978;101(1):83-117.

34. Bancaud J, Brunet-Bourgin F, Chauvel P, et al. Anatomical origin of deja vu and vivid 'memories' in human temporal lobe epilepsy. Brain. 1994;117(Pt 1):71-90

35. Penfield W. The role of temporal cortex in certain psychical phenomena Journal of Mental Science. 1955;101:451-465.

36. Penfield W. Functional localization in temporal and deep Sylvian areas. Research Publications Association for Research in Nervous and Mental Disease. 1958;36:210-226.

37. Neppe VM: A single cause of déjà vu or multiple etiologies? In: Neppe VM \& Funkhouser AT (Eds.), Déjà Vu: A Second Look (Chapter 4). Brainvoyage.com. Seattle, USA.

38. Gloor P, Olivier A, Quensey LF, et al. The role of the limbic system in experiential phenomena of temporal lobe epilepsy. Annals of Neurology . 1981;12(2):129-144.

39. Weinand ME, Hermann B, Wyler AR, et al. Long-term subdural strip electrocorticographic monitoring of ictal deja vu. Epilepsia.994;35(5):1054-1059.

40. Neppe VM. When is déjà vu not déjà vu? A second look at related phenomena. In: Neppe VM \& Funkhouser AT (Eds.), Déjà Vu: A Second Look (Chapter 16b). Brainvoyage.com. Seattle, USA. 2006. p.178-188.

41. Tabet N, Sivaloganathan S. A case of persistent deja vu in an elderly patient. Progress in Neurology and Psychiatry. 2001;5:18-19.

42. Wais PE, Wixted JT, Hopkins RO, et al. The Hippocampus Supports both the Recollection and the Familiarity Components of Recognition Memory. Neuron. 2006;49(3):459-466.

43. Pick A. On reduplicative paramnesia. Brain . 1903;26:260-267.

44. Kopelman MD. Varieties of false memory. Cognitive Neuropsychology. 1999;16:197-214

45. Weinstein EA. Symbolic aspects of confabulation following brain injury: influence of premorbid personality. Bull Menninger Clin. 1996;60(3):331-350.

46. Wigan A. The duality of the mind. Longaan, Brown, Green and Longman, London. 1844. 
47. Maudsley H. The double brain. Mind. 1989;14:160.

48. Grasset J .La sensation déjà vu. Journal de Psychologie Normale et Pathologique. 1904;1:17.

49. Titchener EB. A Beginner's Psychology. McMillan publishers, New York, USA. 1917.

50. Efron R. Temporal perception, aphasia and déjà vu. Brain. 1963;86(3):403-424.

51. Comfort A. Homuncular identity-sense as a deja-vu phenomenon British Journal of Medical Psychology. 1977; 50(4):313-315.

52. Neppe VM. Why parapsychology is amongst the most important of the sciences. Australian J of Parapsychology. 2005;5(1):4-22.

53. Adachi N, Koutroumanidis M, Elwes RD, et al. Interictal 18FDG PET findings in temporal lobe epilepsy with deja vu. J Neuropsychiatry Clin Neurosci. 1999;11(3):380-386.

54. Neppe VM. The modern era of déjà vu research: The Neppe phenomenological research (Section 5). IQNexus Journal. 2005;7(1):32-39.

55. Neppe VM. Ensuring homogeneous data collection for present and future research on possible psi phenomena by detailing subjective descriptions, using the multi-axial A to Z SEATTLE classification. . Neuroquantology . 2011;9(1):84-105.

56. Adachi N, Akanuma N, Adachi T, et al. Deja vu experiences are rarely associated with pathological dissociation. J Nerv Ment Dis. 2008;196(5):417-419.

57. Sno HN, Schalken HF, de Jonghe F, et al. The inventory for deja vu experiences assessment. Development, utility, reliability, and validity. $J$ Nerv Ment Dis. 1994;182(1):27-33.

58. Neppe VM. The New Neppe Déjà Vu Questionnaire-2006. In: Neppe VM \& Funkhouser AT (Eds.), Déjà Vu: A Second Look (Chapter 20). Brainvoyage.com. Seattle, USA. 2006. p. 235-290.

59. Neppe VM. The New Neppe Deja Vu Questionnaire-2006. Brainvoyage. com. Seattle, WA, USA. 2007.

60. Guedj E, Aubert S, McGonigal A, et al. Déjà-vu in temporal lobe epilepsy: Metabolic pattern of cortical involvement in patients with normal brain MRI. Neuropsychologia. 2010;48(7):2174-2181.

61. Neppe VMIs. deja vu a symptom of temporal lobe epilepsy? S Afr Med J. 1981;60(23):907-908.

62. Neppe VM. The different presentations of the deja vu phenomenon: New research. Parapsychological Journal of South Africa. 1983;4(2):124-139.

63. Neppe VM. Deja vu: phenomenological and diagnostic relevance in temporal lobe epilepsy Epilepsia. 1986;27(5):608.

64. Neppe VM. Déjà Vu Revisited. Brainvoyage.com Brainquest Press, Seattle, USA. 2006.

65. Neppe VM. Single cause of déjà vu or multiple etiologies? In: Neppe VM \& Funkhouser AT (Eds.), Déjà Vu: A Second Look (Chapter 4). Brainvoyage.com. Seattle, USA. 2006. p.39-46.

66. Neppe VM. The qualitative aspects of deja vu experience. In: Neppe VM \& Funkhouser AT (Eds.), Déjà Vu Revisited. Brainvoyage.com. Brainquest Press, Seattle, USA. 2006. p. 13-64.

67. Neppe VM, Bradu D. Déjà vu subtypes: four challenges for researchers In: Neppe VM \& Funkhouser AT (Eds.) Déjà Vu: A Second Look. Brainvoyage.com. Seattle, USA. 2006. p. 52-67.
68. Neppe VM. Déjà Vu: Glossary and Library. Seattle: Brainvoyage.com. 2006.

69. Neppe VM, Ellegala D, Baker C. The Inventory of Neppe of Symptoms of Epilepsy and the Temporal Lobe (INSET): A new rating scale. Epilepsia . 1991;32(5):4.

70. Neppe VM. Deja Vu Revisited. Brainvoyage.com. Seattle, USA. 2006.

71. Sno HN, Draaisma D. An early Dutch study of déjà vu experiences. Psychol Med. 1993;23(1):17-26.

72. Neppe VM, Funkhouser AT. Modern research on déjà vu: A brief, selective update of recent ideas. Déjà Vu: A Second Look (Chapter 17). Brianvoyage.com. Seattle, USA. 2006.

73. Brown AS. The déjà vu experience. Psychology Press, New York. 2004

74. Neppe VM. Non-epileptic symptoms of temporal lobe dysfunction. South African Medical Journal. 1981;60(26):989991.

75. Neppe VM. How definitions impact the incidence of déjà vu. Déjà Vu: A Second Look (Chapter 7A). Brianvoyage.com. Seattle, USA. 2006.

76. Lennox WG, Cobb S. Aura in epilepsy: a statistical review of 1,359 cases. Arch NeurPsych. 1933;30(2):374-387.

77. Sengoku A, Toichi M, Murai T. Dreamy states and psychoses in temporal lobe epilepsy: mediating role of affect. Psychiatry Clin Neurosci. 1997;51(1):23-26.

78. Cole M, Zangwill OL. Deja vu in temporal lobe epilepsy. J Neurol Neurosurg Psychiatry. 1963;26:37-38.

79. Funkhouser AT. Déjà vu in Western history: A review. In: Neppe VM \& Funkhouser AT (Eds.), Déjà Vu: A Second Look (Chapter 2). Brainvoyage.com. Seattle, USA. 2006. p. 21-30.

80. Neppe VM, Bradu D. Déjà vu subtypes: four challenges for researchers. In: Neppe VM \& Funkhouser AT (Eds.), Déjà Vu: A Second Look (Chapter 6). Brainvoyage.com. Seattle, USA. 2006.

81. Neppe VM. Déjà vu: An overview of a fascinating subjective experience. IQNexus Journal. 2015;7(1):7-56.

82. Neppe VM. Non-epileptic symptoms of temporal lobe dysfunction $S$ Afr Med J. 1981;60(26):989-991.

83. Neppe VM. Cry the Beloved Mind: A Voyage of Hope. Brainquest Press, Seattle, USA. 1999.

84. Neppe VM. Innovative Psychopharmacotherapy (1st edn), Raven Press, New York, USA. 1989.

85. Neppe VM, Wessels WH. Psychotic toleration of neuroleptic medication. South African Medical Journal. 1979;56(27):1147.

86. Moulin CJ, Conway MA, Thompson RG, et al. Disordered memory awareness: recollective confabulation in two cases of persistent deja vécu. Neuropsychologia. 2005;43(9):1362-1378.

87. Neppe VM, Kaplan C. Short-term treatment of atypical spells with carbamazepine. Clin Neuropharmacol. 1988;11(3):287-289.

88. Petho B. Chronophrenia--a new syndrome in functional psychoses. Psychopathology. 1985;18(2-3):174-180.

89. Neppe VM. When is déjà vu not déjà vu? A second look at paramnesias. In: Neppe VM \& Funkhouser AT (Eds.), Deja Vu A Second Look (Chapter 16A). Brainvoyage.com. Seattle, USA. 2006.

90. Brown AS. The Deja Vu Experience: Essays in cognitive psychology. Psychology Press, New York, USA. 2004. 